\title{
Recruitment of the occipital cortex by arithmetic processing follows computational bias in the congenitally blind
}

\author{
Virginie Crollen $^{\text {a,c, }}$, Latifa Lazzouni ${ }^{\mathrm{b}}$, Mohamed Rezk ${ }^{\mathrm{c}}$, Antoine Bellemare ${ }^{\mathrm{b}}$, Franco Lepore ${ }^{\mathrm{b}}$, \\ Marie-Pascale Noël ${ }^{c}$, Xavier Seron ${ }^{\mathrm{c}}$, Olivier Collignon ${ }^{\mathrm{a}, \mathrm{c}, * *}$ \\ ${ }^{\text {a }}$ Centre for Mind/Brain Science, University of Trento, Via delle regole 101, 38123, Mattarello, TN, Italy \\ ${ }^{\mathrm{b}}$ Centre de Recherche en Neuropsychologie et Cognition (CERNEC), Université de Montréal, Pavillon Marie-Victorin, 90, rue Vincent d'Indy, Montréal, Québec, H2V 2S9, \\ Canada \\ ${ }^{\mathrm{c}}$ Institute of Psychology (IPSY) and Institute of Neuroscience (IoNS), Université Catholique de Louvain, Place Cardinal Mercier 10, 1348, Louvain-la-Neuve, Belgium
}

\section{A R T I C L E I N F O}

\section{Keywords:}

Blindness

Mental arithmetic

Multiplication

Neural correlates

Subtraction

\begin{abstract}
A B S T R A C T
Arithmetic reasoning activates the occipital cortex of congenitally blind people (CB). This activation of visual areas may highlight the functional flexibility of occipital regions deprived of their dominant inputs or relate to the intrinsic computational role of specific occipital regions. We contrasted these competing hypotheses by characterizing the brain activity of $\mathrm{CB}$ and sighted participants while performing subtraction, multiplication and a control letter task. In both groups, subtraction selectively activated a bilateral dorsal network commonly activated during spatial processing. Multiplication triggered activity in temporal regions thought to participate in memory retrieval. No between-group difference was observed for the multiplication task whereas subtraction induced enhanced activity in the right dorsal occipital cortex of the blind individuals only. As this area overlaps with regions showing selective tuning to auditory spatial processing and exhibits increased functional connectivity with a dorsal "spatial" network, our results suggest that the recruitment of occipital regions during high-level cognition in the blind actually relates to the intrinsic computational role of the activated regions.
\end{abstract}

\section{Introduction}

Studies involving congenitally blind people (CB) provide important insights into the role played by experience and intrinsic biological constraints in shaping the development of the sensory and cognitive tuning of brain regions. In $\mathrm{CB}$ individuals, regions of the occipital cortex that typically process visual information massively enhance their response to non-visual inputs (Bavelier and Neville, 2002; Sadato et al., 1996).

It has been suggested that the mechanisms guiding this brain organization process are constrained by the native functional role of the occipital cortex. For example, hMT+/V5, a region chiefly dedicated to processing visual motion in the sighted, preferentially processes auditory (Dormal et al., 2016a; Jiang et al., 2016; Poirier et al., 2006) and tactile motion (Ricciardi et al., 2007) in the blind. Moreover, right dorsal occipital regions typically involved in visuospatial localization in the sighted are active when blind individuals localize sounds (Collignon et al., 2011) and altering the function of this region with transcranial magnetic stimulation (TMS) selectively disrupts auditory localization, but not pitch or sound intensity, in the blind but not the sighted (Collignon et al., 2007). Similarly, the visual word form area (VWFA), a region specialized in processing visual orthographic information in the sighted, seems to be selectively recruited in the blind when processing braille words (Büchel et al., 1998; Reich et al., 2011). These studies suggest that even if the occipital cortex of blind individuals extends its tuning toward non-visual inputs, this tuning is not stochastic but is rather constrained by the intrinsic computational bias of local regions (Collignon et al., 2009, 2012; Heimler et al., 2015; Ricciardi et al., 2014).

Contrasting with this view, it has been suggested that the occipital cortex of CB engages in higher-level cognitive operations that apparently have little to do with occipital functions such as memory (Amedi et al., 2003), language processing (Bedny et al., 2011; Röder et al., 2002) or numerical thinking (Kanjlia et al., 2016). On the basis of these observations, it has been proposed that the human cortex is functionally flexible early in life (Lane et al., 2015) and can adopt a wide range of distant computation depending on experience (Bedny, 2017). However, this latter argument rests on the presupposition that higher-cognitive

\footnotetext{
* Corresponding author. Institute of Psychology (IPSY) and Institute of Neuroscience (IoNS), Université Catholique de Louvain, Belgium.

** Corresponding author. Institute of Psychology (IPSY) and Institute of Neuroscience (IoNS), Université Catholique de Louvain, Belgium.

E-mail addresses: virginie.crollen@uclouvain.be (V. Crollen), olivier.collignon@uclouvain.be (O. Collignon).
} 
functions have no computational relation with vision. But is this the case? For instance, the fact that arithmetic processing recruits occipital regions in $\mathrm{CB}$ has recently been used as a proof-of-principle that the occipital cortex is cognitively pluripotent at birth and capable of assuming a broad range of unrelated cognitive functions apparently distant from visual processes (Kanjlia et al., 2016). However, several studies have suggested that the foundations of some arithmetic operations are rooted in specific visuo-spatial mechanisms (Burr et al., 2018).

Additions and subtractions have been thought to involve shifts of attention along a mental number line: a shift of attention toward the right (or toward larger numbers) for addition and toward the left (smaller numbers) for subtraction (Knops et al., 2009a,b; Masson and Pesenti, 2014; McCrink et al., 2007; Pinhas and Fischer, 2008). Importantly, these shifts of attention have not been reported for multiplication and division operations (Katz and Knops, 2014; Katz et al., 2017, Li et al., 2018; Mathieu et al., 2016). In accordance with this operation dissociation, it has also been demonstrated that adults and even children use a variety of strategies while solving arithmetic operations (Campbell and Timm, 2000; Dehaene and Cohen, 1997; Hecht, 1999; LeFevre et al., 1996; Siegler and Shrager, 1984). While retrieval is the dominant method for multiplications, subtraction principally relies on quantity manipulation procedures (Campbell and Xue, 2001). Interestingly, these differential procedures might play an important role in shaping the neural foundations of arithmetic (Chochon et al., 1999; Dehaene and Cohen, 1997; Delazer and Benke, 1997; Lee, 2000; Menon et al., 2000; Pesenti et al., 1994; van Harskamp and Cipolotti, 2001; Zago et al., 2001; Zhou et al., 2007). It has for example been shown that the resolution of subtraction principally engages a network of dorsal parieto-frontal regions (Chochon et al., 1999; Mathieu et al., 2018a,b; Piazza et al., 2007; Prado et al., 2011, 2014) presumably due to the "spatial" strategies used to solve such operations (Siegler and Shrager, 1984). Like subtraction, multiplication requires the mental manipulation of symbolic numbers, yet it is believed to rely on a distinct temporo-parietal network (Chochon et al., 1999; Mathieu et al., 2018a,b; Prado et al., 2011, 2014, Zhou et al., 2007) presumably because multiplications are solved by direct fact retrieval (Cooney et al., 1988).

The present study was designed to test whether the recruitment of the occipital cortex by higher-level cognitive functions in $\mathrm{CB}$ individuals depends on the intrinsic computational role of specific regions. The study of arithmetic processing represents an interesting testbed to help disentangle the hypotheses regarding the mechanisms governing brain (re)organization in CB (functional recycling versus pluripotency). Indeed, as already mentioned, separate arithmetic operations rely on separate brain networks depending on the computational principles they rely on. This raises the question of whether these different operations produce equivalent neural responses in the occipital cortex of blind individuals, or if, as we presume, subtraction will find a privileged neural niche in dorsal occipital regions since these regions have a privileged role in processing spatial relationships in the CB (Dormal et al., 2012).

Moreover, we tested whether the specific regions presumably showing differences of activation between blind and sighted people would show a specific connectivity profile, extracted from independent resting state measurements, potentially supporting the idea that the selective recruitment of specific occipital regions in the blind links to a connectivity profile with functionally selective networks outside occipital regions (Abboud et al., 2015; Hannagan et al., 2015).

We therefore examined the brain activity of $14 \mathrm{CB}$ and 16 sighted participants while they were verifying the results of subtractions and multiplications. If the occipital cortex of CB people is similarly activated for both arithmetic operations, this would support the idea that occipital regions are functionally flexible during development and can adopt a wide range of computation depending on experience (Bedny, 2017). Alternatively, if solving subtraction problems (which in contrast to multiplication relies on spatial strategies) specifically engages, in the blind, regions of the dorsal occipital cortex typically involved in visuo-spatial processing and typically connected to a dorsal parieto-frontal network, this would support the idea that the take-over of occipital regions by higher cognitive functions in the blind actually relates to the intrinsic computation of the regions.

\section{Materials and methods}

\subsection{Participants}

Sixteen sighted controls (SC) [6 females, age range 22-64y, (mean \pm SD, $44 \pm 14 \mathrm{y}$ )] and $14 \mathrm{CB}$ participants [3 females, age range 23-61 y, (mean \pm SD, $44 \pm 13$ y)] took part in the study (see Supplemental Table 1 for a detailed description of the CB participants). The SC did not statistically differ from the CB group in age $(\mathrm{t}(28)=-0.02$, $\mathrm{p}>0.9)$ and sex ratio $(\chi 2=1.54, \mathrm{p}=0.21)$. The participants in the blind group were totally blind since birth or had, at the most, only rudimentary sensitivity for brightness differences and never experienced patterned vision (never saw colors, shapes, or motion). In all cases, blindness was attributed to peripheral deficits with no additional neurological problems. Procedures were approved by the Research Ethics Boards of the University of Montreal. Experiments were undertaken with the understanding and written consent of each participant. Sighted participants were blindfolded when performing the task.

\subsection{Task and general experimental design}

Participants were scanned in 1 fMRI session using a block design procedure. During scanning, participants had to: 1) verify the result of subtractions; 2) verify the result of multiplications; and 3) perform vowel/consonant judgment verification on letters. Additions were not presented as they are thought to not only involve spatial displacements on the mental number line but are also assumed to rely on rote verbal memory (Dehaene and Cohen, 1997). In the subtraction verification task, triplets of auditory numbers were presented and participants had to judge whether the third number corresponded to the difference of the first two numbers. The first operand was either 11 or 13; the second operand ranged from 3 to 8 . The third number was either the correct result of the subtraction or the correct result \pm 1 . In the multiplication verification task, triplets of auditory numbers were presented and participants had to judge whether the third number corresponded to the product of the first 2 numbers. The first operand was either 3 or 4 . The second operand ranged from 3 to 8 . The third number presented corresponded either to the correct result or to the correct result \pm the first operand (e.g., three, five, twelve). In order to examine the neural activity of arithmetic in general (common activity for subtraction and multiplication), participants also had to perform a control letter task. This task was matched to the numerical tasks in terms of stimulus presentation (3 consecutive - non-numerical - symbolic stimuli) and response requirements. Triplets of letters were thus auditorily presented and participants had to judge whether the third letter pertained to the same category (vowel vs. consonant) as the first 2 letters (the first 2 letters were always of the same category). The letters used were the vowels A, E, I, O, U and the consonants B, D, M, N, P, R, S. Participants responded with their right index finger using the top (for correct triplet) or the bottom (for incorrect triplet) key of a response box.

The fMRI session consisted of 30 successive blocks (24s duration each) alternating the three tasks in a fixed order and separated by rest periods ranging from 7 to $9 \mathrm{~s}$ (median $8 \mathrm{~s}$ ). Each block consisted of 6 successive auditory triplets of $4000 \mathrm{~ms}$. In the scanner, auditory stimuli were delivered by means of circumaural fMRI-compatible headphones (Mr Confon, Magdeburg, Germany).

Before the fMRI acquisition, all participants underwent a training session in a mock scanner with recorded scanner noise played in the bore of the stimulator to familiarize them with the fMRI environment and to ensure that the participants understood the task. 


\section{3. fMRI data acquisition and analyses}

Functional MRI-series were acquired using a 3-T TRIO TIM system (Siemens, Erlangen, Germany), equipped with a 12-channel head coil. Multislice T2*-weighted fMRI images were obtained with a gradient echoplanar sequence using axial slice orientation $(\mathrm{TR}=2200 \mathrm{~ms}$, $\mathrm{TE}=30 \mathrm{~ms}, \mathrm{FA}=90^{\circ}, 35$ transverse slices, $3.2 \mathrm{~mm}$ slice thickness, $0.8 \mathrm{~mm}$ inter-slice gap, $\mathrm{FoV}=192 \times 192 \mathrm{~mm}^{2}$, matrix size $=64 \times 64 \times 35$, voxel size $=3 \times 3 \times 3.2 \mathrm{~mm}^{3}$ ). Slices were sequentially acquired along the z-axis in feet-to-head direction. Sequential slice acquisition was chosen because it eases slice timing correction, which in turns helps more reliable connectivity measurement.

The 4 initial scans were discarded to allow for steady state magnetization. Participants' heads were immobilized with the use of foam pads that applied pressure onto the headphones. A structural T1-weighted 3D MP-RAGE sequence (voxel size $=1 \times 1 \times 1.2 \mathrm{~mm}^{3}$; matrix size $=240 \times 256 ; \mathrm{TR}=2300 \mathrm{~ms}, \mathrm{TE}=2.91 \mathrm{~ms}, \mathrm{TI}=900 \mathrm{~ms}, \mathrm{FoV}=256$; 160 slices) was also acquired for all participants.

Functional volumes were pre-processed and analyzed using SPM8 (http://www.fil.ion.ucl.ac.uk/spm/software/spm8/; Wellcome Department of Imaging Neuroscience, London), implemented in MATLAB (MathWorks). Pre-processing included slice timing correction of the functional time series (Sladky et al., 2011), realignment of functional time series, co-registration of functional and anatomical data, a spatial normalization to an echo planar imaging template conforming to the Montreal Neurological institute space, and a spatial smoothing (Gaussian kernel, $8 \mathrm{~mm}$ full-width at half-maximum, FWHM).

Following pre-processing steps, the analysis of fMRI data, based on a mixed effects model, was conducted in two serial steps accounting respectively for fixed and random effects. For each subject, changes in brain regional responses were estimated through a general linear model including the responses to the 3 experimental conditions (subtractions, multiplications, letters). These regressors consisted of a boxcar function convolved with a canonical double-gamma hemodynamic response function. Movement parameters derived from realignment of the functional volumes (translations in $\mathrm{x}, \mathrm{y}$ and $\mathrm{z}$ directions and rotations around $\mathrm{x}, \mathrm{y}$ and $\mathrm{z}$ axes) and a constant vector were also included as covariates of no interest. High-pass filtering was implemented in the design matrix using a cut-off period of $128 \mathrm{~s}$ to remove slow drifts from the time series. Linear contrasts tested the main effect of each condition ([Subtraction], [Multiplication], [Letter]), the main effect of arithmetic ([Subtraction $\cap$ Multiplication $>$ Letter]), the main effect of the Subtraction condition ([Subtraction $>$ Multiplication]) and the main effect of the multiplication condition ([Multiplication $>$ Subtraction]). A balanced contrast was used to test the effect of arithmetic [0.5 (Subtraction)/0.5 (Multiplication)/ -1 (Letter)]. These linear contrasts generated statistical parametric maps [SPM(T)]. The resulting contrast images were then further spatially smoothed (Gaussian kernel $8 \mathrm{~mm}$ FWHM) and entered in a second-level analysis, corresponding to a random effects model, accounting for intersubject variance. The combination of the 2 smoothing operations (at the first and second-level analysis) is equivalent to applying a single smoothing kernel with $11.3 \mathrm{~mm}$ FWHM (given by the square root of the sum of squares of the two smoothing parameters: $\operatorname{sqrt}(82+82)$ ). Such settings are quite classical/adequate (Penny et al., 2008) when someone does not wish (like us here) to disentangle between differential activity in small (in size) and in closely adjacent brain areas. In such a case, smoothing has the main advantages of (1) blurring acquisition noise at an intra-subject level, (2) blurring the important inter-individual anatomical differences when pooling subjects at the second-level random-effect analysis step, and (3) providing an adequate but less constraining ground for performing appropriate corrected statistics.

For each contrast, one-sample t-tests were carried out in each group separately. Two-sample t-tests were then computed to identify group differences for each separate contrast. Group effects [Blind $>$ Sighted] and [Sighted $>$ Blind] were inclusively masked $(\mathrm{p}<0.001$ uncorrected for multiple comparisons) by the main effect in the blind and the sighted group, respectively. Statistical inferences were performed at a threshold of $\mathrm{p}<0.05$ after correction for multiple comparisons (Family Wise Error method) over the entire brain volume or over small spherical volumes ( $10 \mathrm{~mm}$ radius) located in regions of interest (SVC). To select the coordinates of interest, we consulted a body of literature examining brain activations related to numerical processing in sighted individuals and related to brain organization in the blind. Before performing any smallvolume correction (SVC), peaks reported in Talairach space (Talairach and Tournoux, 1988) were transformed to Montreal Neurological Institute space using Matthew Brett's bilinear transformation (http:// imaging.mrc-cbu.cam.ac.uk/imaging/MniTalairach). Standard stereotactic coordinates $(\mathrm{x}, \mathrm{y}, \mathrm{z})$ used for SVC are listed in supplemental material (in MNI space). Our results were finally compared to the results of a previous study investigating the brain activity of $\mathrm{CB}$ and $\mathrm{SC}$ in processing either the spatial or the pitch properties of sounds (Collignon et al., 2011).

\subsection{Resting-state functional connectivity analysis}

To control for possible motion artifacts, a scrubbing approach was implemented using Artifact detection tools (ART; www.nitrc.org/ projects/artifact_detect) to identify outlier volumes that had a difference in scan-to-scan global intensity more than 9 standard deviations away from the mean global brain signal, or volumes that had more than $2 \mathrm{~mm}$ of scan-to-scan composite motion. The outlier time points were used as a first level covariate. Afterwards, the functional volumes were spatially smoothed with a 4-mm full-width half-maximum (FWHM) Gaussian kernel. To rule out the possibility that any connectivity changes could be attributed to motion (Power et al., 2012; Satterthwaite et al., 2012; Van Dijk et al., 2012), we compared the average and maximum motion between groups using a two-sample $t$-test, and found no significant difference between the 2 groups in the average $(\mathrm{t}(22)=0.35$, $\mathrm{p}=0.73$ ) or the maximum motion $(\mathrm{t}(22)=0.67, \mathrm{p}=0.508)$.

Functional connectivity analysis was performed in the CONN functional connectivity toolbox (Whitfield-Gabrieli and Nieto-Castanon, 2012); http://www.nitrc.org/projects/conn). A component-based noise correction (Compcorr) (Behzadi et al., 2007) strategy was implemented to control for nuisance effects and physiological noise. Motion parameters and their first derivatives as well as outlier time points from the ART toolbox were included as first level covariates to remove the variance related to head motion. Linear de-trending and a band-pass filter of $0.008-0.09 \mathrm{~Hz}$ were applied to the functional data. First level analysis was performed in the CONN framework to investigate functional connectivity changes within-subject. Pearson's correlation coefficient was used as a measure of functional connectivity. A seed-based correlation analysis using a voxel-wise approach was performed. We used the left and right middle occipital gyri as our seed regions because these regions showed activity for the contrasts $[\mathrm{CB}>\mathrm{SC}][$ Subtraction $\cap$ Multiplication $>$ Letter] and [CB $>$ SC] [Subtraction $>$ Multiplication] - see results section.

The time series from each seed region was extracted by averaging the signal from all the voxels in the seed ROI. The time series from each seed was then correlated with each voxel in the rest of the brain. The correlation coefficients were Fisher-transformed to perform second-level statistical comparisons across groups. A one-sided two-samples $t$-test was performed to assess differences in the functional connectivity between groups $[\mathrm{CB}>\mathrm{SC}$ ]. Results were thresholded at $\mathrm{p}<0.001$ at the voxel level and FDR-corrected $\mathrm{p}<0.05$ at the cluster level.

\section{Results}

\subsection{Behavioral results}

Participants' performances were analyzed with a 3 (experimental conditions: subtraction, multiplication, letter) x 2 (groups: SC vs. $\mathrm{CB}$ ) repeated measures ANOVA performed on the percentage of correct responses. This analysis showed a marginal effect of condition, $\mathrm{F}(2,56)=3.13, \mathrm{p}=0.05$, 
$\eta \mathrm{P} 2=0.10$. Both groups tended to be less efficient in the subtraction condition $(\mathrm{M} \pm \mathrm{SE}=87.07 \pm 2.55)$ than in the 2 other conditions $(\mathrm{M} \pm \mathrm{SE}=91.38 \pm 1.29$ for multiplication; $\mathrm{M} \pm \mathrm{SE}=91.97 \pm 1.80$ for letters). The group effect was not significant, $\mathrm{F}(1,28)=0.09, \mathrm{p}=0.76$, $\eta \mathrm{P} 2=0.003$, nor was the group $\times$ condition interaction, $\mathrm{F}(2,56)=0.76$, $\mathrm{p}=0.47, \eta \mathrm{P} 2=0.03$ (see Fig. $1 \mathrm{j}$ ).

\section{2. $f M R I$ results}

\subsubsection{General arithmetic}

A conjunction analysis performed across groups disclosed arithmetic selectivity in the left inferior parietal lobule (IPL) for both groups. To investigate the effect of congenital blindness on global arithmetic processing, we compared the cerebral responses of blind vs. sighted participants for both multiplication and subtraction relative to the letter condition ([CB $>\mathrm{SC}$ ] [subtraction $\cap$ multiplication $>$ letter]). This analysis yielded significant results in the left posterior lateral occipital area (hOC4lp) (see Fig. 1 and Table 1). The opposite contrast ([SC $>$ CB] [subtraction $\cap$ multiplication $>$ letter]) did not yield any significant effect.

\subsubsection{Subtraction}

The middle frontal gyrus (MFG) and the superior parietal lobule (PSPL)/intraparietal sulcus (IPS) demonstrated enhanced activity for subtraction over multiplication in both groups of participants ([SC $\cap \mathrm{CB}]$ [subtraction $>$ multiplication]). Crucially, the right hOC4lp demonstrated enhanced activity in $\mathrm{CB}$ when compared to SC ([CB $>\mathrm{SC}$ ] [subtraction $>$ multiplication]). In general, deactivation of this region was found in the sighted while activation was observed in CB (see Fig. 1 and Table 1).

The opposite contrast ([SC $>$ CB] [subtraction $>$ multiplication]) did not yield any significant effect.

In a previous study comparing the brain activity of $\mathrm{CB}$ and SC in processing either the spatial or the pitch properties of sounds, Collignon et al. (2011) demonstrated that auditory-spatial processing mainly recruits the right cuneus and the right middle occipital gyrus in the blind. These two regions of the dorsal occipital stream are known to be involved in visuospatial/motion processing in sighted and, as shown in Supplemental Fig. 1, partly overlap the region showing a subtraction-specific activation in the current experiment.
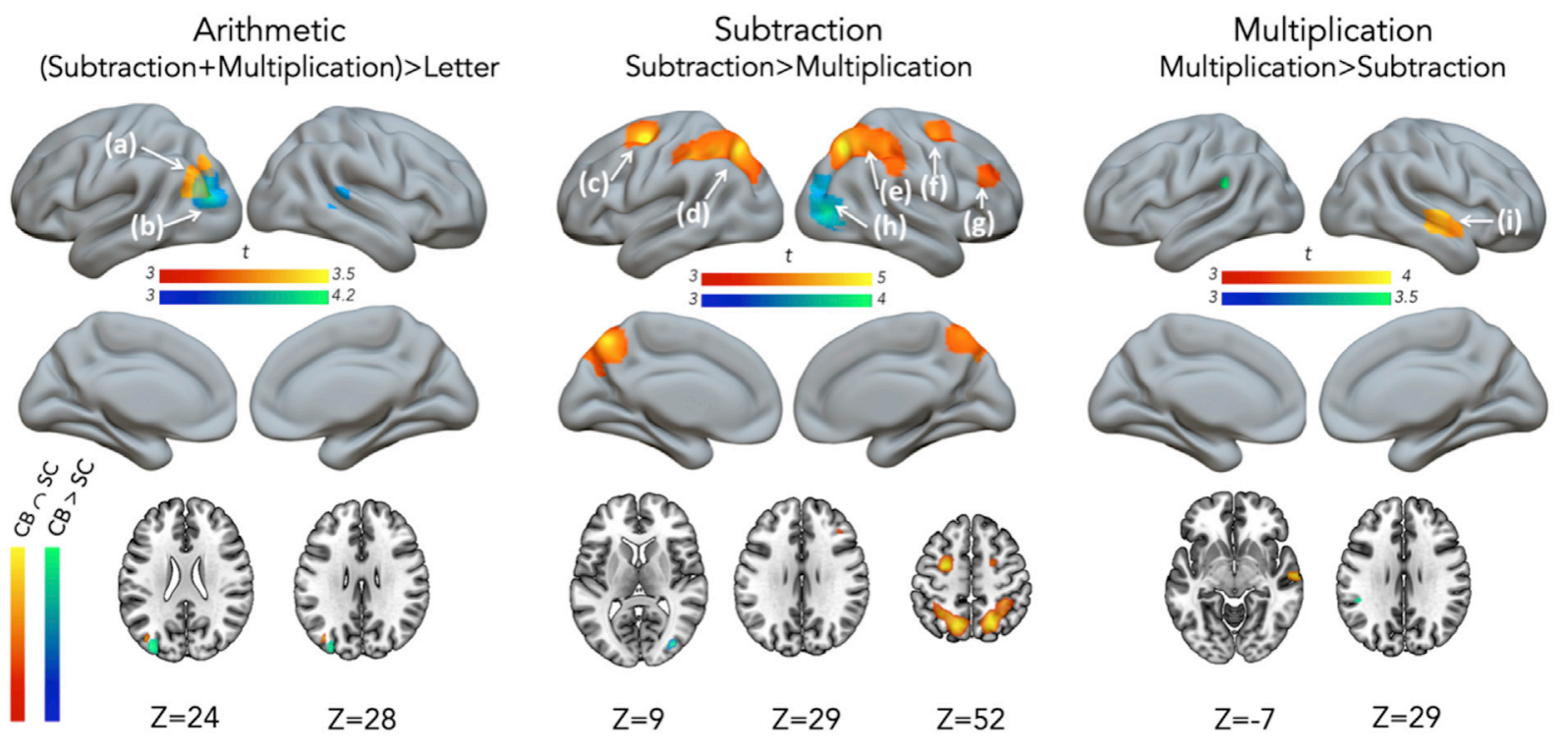

(a) L Inferior parietal lobule

(b) L hOC4Ip

(c) L Middle Frontal Gyrus

(d) L SPL \& IPS

(e) R SPL \& IPS
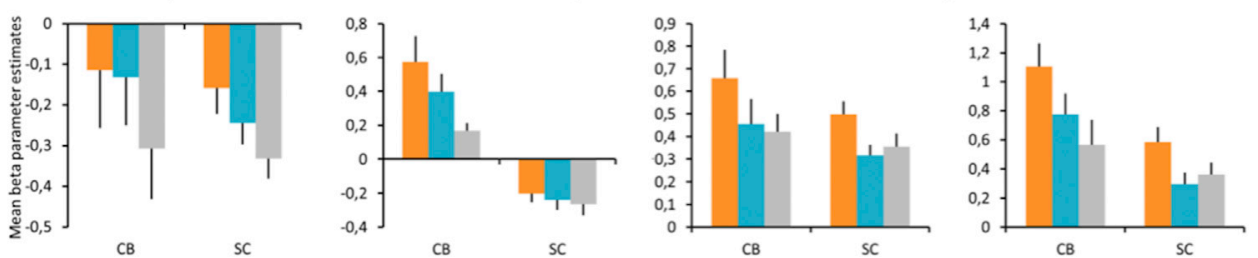

(f) R Middle Frontal Gyrus (g) R Middle Frontal Gyrus (h) R hOC4lp
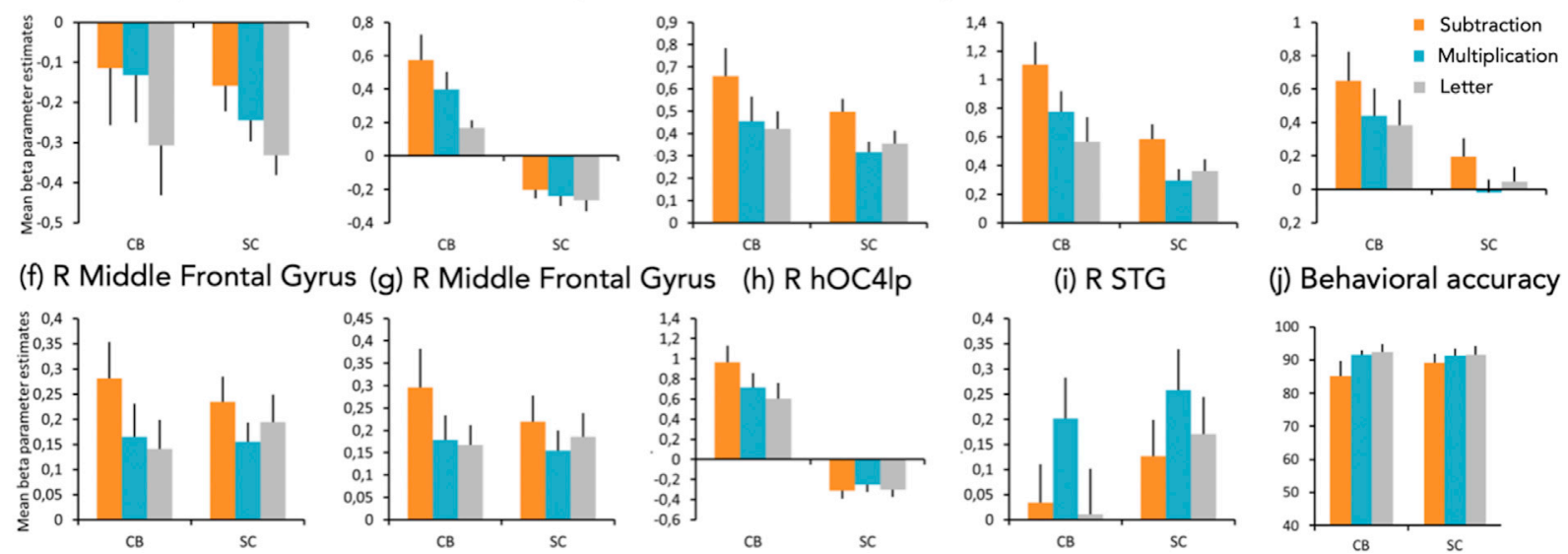

(j) Behavioral accuracy

Fig. 1. Results of the whole brain univariate analyses. Upper part: Task-related brain activity common to both groups in orange ( $p$ uncorr $<0.002, \mathrm{k}>15)$ and specific to the blind group in blue ( $\mathrm{p}$ uncorr $<0.001, \mathrm{k}>15$ ). There were no activations specific to the sighted group. Color bars represent $\mathrm{t}$-values. Lower part (a-i): for illustration, mean activity estimates (arbitrary units \pm SEM) associated with subtraction (in orange), multiplication (in blue) and letter (in grey) are plotted for blind and sighted at significant peaks. See Table 1 for a list of brain regions depicted in this figure. (j) Behavioral results. Mean and standard error of accuracy scores (percentage of correct responses) per condition and group. 
Table 1

Functional results summarizing the main effect of groups for the different contrasts of interests.

\begin{tabular}{|c|c|c|c|c|c|c|}
\hline Area & Cluster Size $(\Phi)$ & $\mathrm{x}$ & $\mathrm{y}$ & $\mathrm{z}$ & $\mathrm{Z}$ & $\mathrm{p}$ \\
\hline \multicolumn{7}{|c|}{ (A) [SC $\cap \mathrm{CB}$ ] [Subtraction $\cap$ Multiplication $>$ Letter] } \\
\hline L IPL & 89 & -40 & -74 & 26 & 3.03 & $0.02^{*}$ \\
\hline \multicolumn{7}{|c|}{ (B) $[\mathrm{CB}>\mathrm{SC}]$ [Subtraction $\cap$ Multiplication $>$ Letter] } \\
\hline L hOC4lp & 321 & -34 & -86 & 24 & 3.98 & $0.001 *$ \\
\hline \multicolumn{7}{|c|}{ (C) $[\mathrm{SC} \cap \mathrm{CB}]$ [Subtraction $>$ Multiplication] } \\
\hline L MFG & 426 & -26 & -2 & 54 & 2.63 & $0.04^{\#}$ \\
\hline R MFG & 124 & 22 & -2 & 56 & 3.66 & $0.004 *$ \\
\hline L SPL & 2818 & -20 & -68 & 46 & 4.06 & $0.001 *$ \\
\hline R SPL & & 24 & -68 & 46 & 4.10 & $0.001^{*}$ \\
\hline R MFG & 53 & 32 & 36 & 24 & 3.25 & $0.01 *$ \\
\hline \multicolumn{7}{|c|}{ (D) $[\mathrm{CB}>\mathrm{SC}]$ [Subtraction $>$ Multiplication] } \\
\hline R hOC4lp & 156 & 36 & -82 & 8 & 3.55 & $0.006^{*}$ \\
\hline \multicolumn{7}{|c|}{ (E) $[\mathrm{SC} \cap \mathrm{CB}]$ [Multiplication $>$ Subtraction] } \\
\hline R STG & 104 & 64 & -14 & -8 & 3.48 & $0.007^{*}$ \\
\hline
\end{tabular}

Brain activations significant (pcorr $<.05$ FWE) after correction over (*) small spherical volumes (SVC, $10 \mathrm{~mm}$ radius). To be significant in contrast $\mathrm{C}$, p-values had to be smaller than 0.01 (correction applied for multiple comparisons). $(\Phi)$ Number of voxels in specific clusters when displayed at p(uncorr) $<0.001$ SC: sighted controls, CB: congenitally blind, L: left, R: Right, IPL: inferior parietal lobule; hOC4lp: posterior lateral occipital area; MFG: middle frontal gyrus; SPL: Superior Parietal Lobule; STG: superior temporal gyrus.

\subsubsection{Multiplication}

There was common multiplication related activity in SC and CB ([SC $\cap \mathrm{CB}$ ] [multiplication $>$ subtraction]) in the right superior temporal gyrus (STG) (see Fig. 1 and Table 1). Neither the contrast ([CB $>$ SC] [multiplication $>$ subtraction]), nor the contrast ([SC $>\mathrm{CB}$ ] [multiplication $>$ subtraction]) yielded significant effects.

To guarantee that the general differences in brain activity between subtraction and multiplication was not merely due to differences in difficulty level, the same analyses as the one described above were also performed by adding accuracy differences between the targeted contrast as a covariate (subtraction accuracy - multiplication accuracy for the contrasts [Subtraction $>$ Multiplication] and ([Multiplication $>$ Subtraction]; mean of subtraction and multiplication accuracy - letter accuracy for the contrast [Subtraction $\cap$ Multiplication $>$ Letter]). As shown in Supplemental Fig. 1, these analyses yielded similar activation maps as the one represented in Fig. 1.

\subsubsection{Functional connectivity}

We found greater correlations between the left hOC4lp and a network of dorsal regions in blind relative to sighted participants (main effect of group, seed to whole-cortex analysis $\mathrm{P}<0.05$, FDR corrected; Fig. 2 and Table 2). A similar pattern was observed for the right hOC4lp $(\mathrm{P}<0.05$, FDR corrected; Fig. 2 and Table 2). Moreover, both seed regions showed enhanced intra-occipital connectivity with ventral occipito-temporal regions as previously shown (Burton et al., 2014; Pelland et al., 2017).

\section{Discussion}

We examined how the lack of visual experience impacts the neural basis of specific arithmetical operations by contrasting activity maps elicited by the execution of subtraction or multiplication operations in $\mathrm{CB}$ and sighted controls.

Activation of occipital regions in the case of congenital blindness provides a unique model for understanding how intrinsic organization and experience together determine cortical function. Previous studies have found that the "visual" cortex of CB people responds to a variety of auditory (Collignon et al., 2009; Gougoux et al., 2005; Ricciardi et al., 2014; Weeks et al., 2000) and tactile tasks (Büchel et al., 1998; Sadato et al., 1996). Several studies have shown that such a phenomenon follows the functional specialization of the colonized brain regions (Amedi et al., 2017; Bi et al., 2016; Cecchetti et al., 2016; Dormal and Collignon, 2011; Heimler et al., 2015). However, the observation that the occipital cortex
Seed-based resing-state functional connectivity: Blind > Sighted
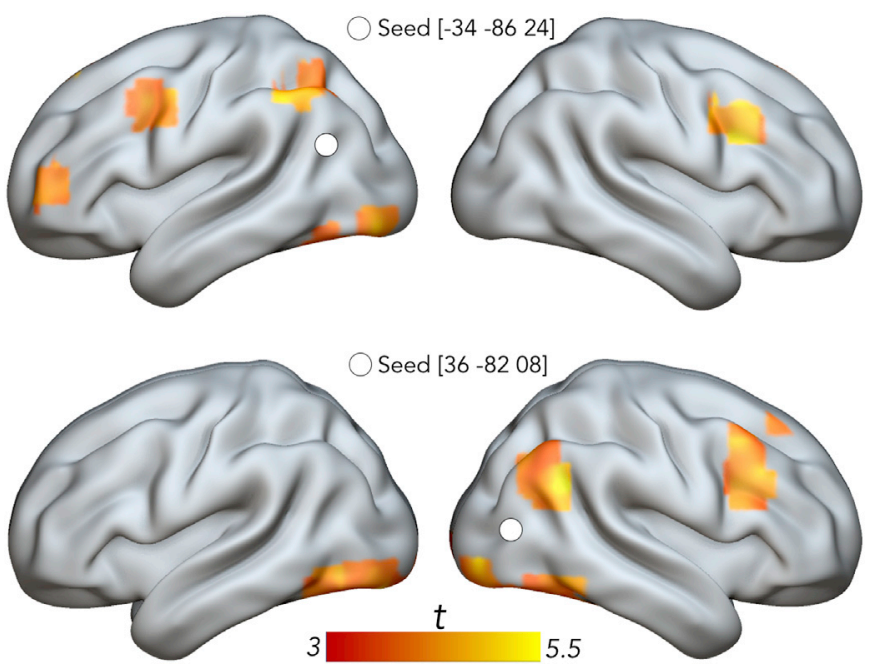

Fig. 2. Brain regions more correlated with left and right hOC4lp in blind $(\mathrm{n}=13)$ relative to sighted $(\mathrm{n}=11)$ individuals in resting-state data $(\mathrm{P}<0.05$, FDR corrected). Left and right hOC4lp seeds shown in white.

Table 2

Brain regions correlating with left and right hOC4lp at rest.

\begin{tabular}{|c|c|c|c|c|c|}
\hline Area & $\begin{array}{l}\text { Cluster } \\
\text { Size }(\Phi)\end{array}$ & $\mathrm{x}$ & $\mathrm{y}$ & $\mathrm{z}$ & $\begin{array}{l}\text { Cluster } \\
\mathrm{P}_{\mathrm{FDR}}\end{array}$ \\
\hline \multicolumn{6}{|c|}{ Correlation with L hOC4lp in blind $>$ sighted } \\
\hline Lingual gyrus & 176 & 0 & -34 & 42 & 0.0001 \\
\hline $\mathrm{L}$ angular gyrus & 151 & -50 & -52 & 48 & 0.0002 \\
\hline L cingulate gyrus & 142 & -6 & 22 & 46 & 0.0003 \\
\hline $\begin{array}{l}\mathrm{R} \text { inferior precentral sulcus } \\
\text { (premotor cortex) }\end{array}$ & 115 & 54 & 8 & 40 & 0.0009 \\
\hline $\mathrm{R}$ retrospenial cortex & 105 & 10 & -46 & 18 & 0.0014 \\
\hline L fusiform gyrus & 78 & -32 & -68 & -12 & 0.0063 \\
\hline L middle frontal gyrus & 75 & -38 & 8 & 40 & 0.0063 \\
\hline L inferior frontal gyrus & 75 & -44 & 46 & 6 & 0.0063 \\
\hline $\mathrm{L}$ thalamus & 73 & -8 & -16 & 10 & 0.0064 \\
\hline L occipito-temporal cortex & 59 & -20 & -46 & -8 & 0.0158 \\
\hline L IPS & 51 & -32 & 8 & 28 & 0.0242 \\
\hline L posterior collateral sulcus & 51 & -24 & -68 & -8 & 0.0242 \\
\hline L inferior occipital gyrus & 50 & -38 & -86 & -12 & 0.0242 \\
\hline Posterior cingulate & 41 & -2 & -40 & 24 & 0.0468 \\
\hline \multicolumn{6}{|c|}{ Correlation with R hOC4lp in blind $>$ sighted } \\
\hline $\mathrm{R}$ inferior parietal lobule & 213 & 48 & -62 & 28 & 0.0001 \\
\hline L cerebellum crus 2 & 207 & -8 & -80 & -32 & 0.0001 \\
\hline $\begin{array}{l}\mathrm{L} \text { precuneus - posterior } \\
\text { cingulate }\end{array}$ & 184 & -2 & -44 & 10 & 0.0001 \\
\hline L inferior occipital gyrus & 127 & -30 & -86 & -18 & 0.0004 \\
\hline $\begin{array}{l}\mathrm{R} \text { middle/inferior frontal } \\
\text { gyrus }\end{array}$ & 86 & 46 & 20 & 30 & 0.0039 \\
\hline $\mathrm{R}$ lingual gyrus & 85 & 12 & -92 & 0 & 0.0039 \\
\hline $\mathrm{R}$ inferior occipital gyrus & 80 & 34 & -92 & -8 & 0.0047 \\
\hline $\mathrm{R}$ middle frontal gyrus & 78 & 48 & 16 & 46 & 0.0047 \\
\hline R fusiform gyrus & 76 & 34 & -68 & -14 & 0.0048 \\
\hline L inferior LOC & 61 & -44 & -82 & -14 & 0.0117 \\
\hline $\mathrm{R}$ inferior temporal cortex & 61 & 34 & -46 & -18 & 0.0117 \\
\hline R superior frontal gyrus & 55 & 30 & 32 & 54 & 0.0145 \\
\hline $\mathrm{R}$ fusiform gyrus & 55 & 54 & -62 & -18 & 0.0145 \\
\hline L fusiform gyrus & 55 & -48 & -62 & -18 & 0.0144 \\
\hline L cerebellum/posterior lobe & 45 & -26 & -62 & -32 & 0.0301 \\
\hline $\mathrm{L}$ thalamus & 39 & -8 & -10 & 6 & 0.0470 \\
\hline
\end{tabular}

of the $\mathrm{CB}$ also activates during higher cognitive operations considered distant from visual functions, such as arithmetic processing (Kanjlia et al., 2016), was used to challenge the idea that an intrinsic computational bias is a generic principle guiding the recruitment of the occipital cortex and that, in contrast, occipital regions are pluripotent early in development and able to engage in a vast array of cognitive functions that 
are evolutionarily and cognitively distant from vision (Bedny, 2017).

Our observation that a specific arithmetic operation - subtraction but not multiplication - triggers enhanced activity in selective occipital regions - the right dorsal stream - provides a bridge between these two apparently incompatible views of occipital activation in CB people. Our findings suggest that the activation of occipital regions for higher cognitive operations may not differ significantly from its original function but may rather emerge from intrinsic computational bias. More precisely, we suggest that the specific recruitment of dorsal occipital regions for subtraction is a by-product of the intrinsic role of this region in spatial processing (Knops et al., 2009a, 2009b; Masson and Pesenti, 2014; McCrink et al., 2007; Pinhas and Fischer, 2008). Supporting this idea, the region showing selective activation for subtraction in our blind population overlapped in part with regions showing preferential tuning to auditory spatial processing in the blind (Collignon et al., 2011; see Supplemental Fig. 2).

It has been proposed that specific numerical processing systematically maps onto parietal circuits because this culturally new invention is based on, or recycles, more 'basic-primitive' cognitive skills, such as space perception or body manipulation, which are older in terms of evolution (Dehaene and Cohen, 2007). We propose that a similar mechanism of functionally specific cortical recycling operates at the ontogenetic level due to the specific experience of blindness. Here, subtraction may selectively remap onto dorsal occipital regions due to its reliance on space processing, which is known to be preserved in these regions in $\mathrm{CB}$ individuals (Dormal et al., 2012). We therefore propose that the recruitment of occipital regions in the blind, even for higher cognitive abilities, finds its "neural niche" in a set of circuits that are sufficiently close to the required function and sufficiently plastic so as to strengthen or reorient a significant proportion of their neural resources for the non-visual function (Collignon et al., 2009). Interestingly, this raises the possibility that other higher cognitive domains such as language, which is also known to remap in the occipital cortex of the blind (Bedny et al., 2011; Röder et al., 2002), are also grounded in the native computational predisposition of these regions (Hasson et al., 2016; Lacey et al., 2012, 2017; van Ackeren et al., 2017). However, as activation per se does not necessarily imply a functional use (Lasne et al., 2018), future studies are needed to further explore and directly test this hypothesis.

What could be the force guiding this functional activation of the occipital cortex? As illustrated by our connectivity analyses (see Fig. 2), the right dorsal occipital region showing enhanced preferential involvement for subtraction in $\mathrm{CB}$ also shows enhanced connectivity with a right dorsal network typically involved in spatial processing and attention (Corbetta et al., 1995; Yantis and Serences, 2003). This result supports the idea that the connectivity profile between brain regions may guide the functional specialization of brain regions and, by extension, constrain how neural activity is expressed in the occipital cortex of the blind (Hannagan et al., 2015). In the context of our study, the reinforcement of a privileged connection between dorsal occipital regions and the intraparietal sulcus, probably rooted in their common involvement in spatial computation, will extend the preserved function of the parietal cortex (eg. for subtraction) toward dorsal occipital regions in the case of early visual deprivation.

This additional involvement of dorsal occipital regions for subtraction in CB inevitably raises the question of what is happening to the network typically involved in this function. Even if in our study we did not find any enhanced activity in sighted versus blind participants, previous studies have suggested that the enhanced occipital involvement in $\mathrm{CB}$ may be concomitant with a reduction of the computational load of the typical network (Dormal et al., 2016a; Mattioni et al., 2018)).

The left IPL was the unique region in which both subtraction and multiplication produced greater activity than the letter control task in both sighted and blind individuals (Fig. 1). Specifically, this region was less deactivated during calculation than when processing letters in both groups. Deactivation has been consistently reported during arithmetic tasks (Grabner et al., 2007; Mizuhara et al., 2005; Rickard et al., 2000;
Wu et al., 2009; Zhou et al., 2007) and has been shown to be negatively correlated to mathematical performance: the stronger the deactivation, the lower the arithmetic performance (Wu et al., 2009).

More importantly, we demonstrated that specific arithmetic operations activate selective networks: subtraction preferentially activated the MFG and the SPL regions while the right STG was activated for multiplication in both groups. This observation is probably not related to the difficulty level of the arithmetic operations presented as the same result was highlighted when difficulty was controlled by being entered as a covariate in the analyses (this is also true for the enhanced activity in right MOG in $\mathrm{CB}$ ). The dissociation between parieto-frontal regions for subtraction and superior temporal regions for multiplication is moreover well in line with the idea that arithmetic is processed in different formats within distinct cerebral pathways (Dehaene and Cohen, 1997). In spite of this selective enhancement of right dorsal occipital regions for subtraction in $\mathrm{CB}$, several regions showed an overlap of activity between groups for arithmetic processing, like the intraparietal sulcus when solving subtraction (Kanjlia et al., 2016). Does this overlap of the brain circuits involved in arithmetic processing in $\mathrm{CB}$ and sighted individuals mean that the way these regions implement numerical processing is immune to visual input and experience? One possibility is that those regions supporting specific numerical operations that are not affected by visual experience rely on computational procedures, such as memory retrieval for multiplication and spatial processing for subtraction (Campbell and Xue, 2001). These procedures may be abstracted from sensory input and experience and may therefore rely on an amodal representational format (Damarla et al., 2016; Eger et al., 2003; Nieder, 2012, 2016). However, the fact that similar regions activate in blind and sighted individuals during the same specific task (i.e., parietal network during subtraction) does not guarantee that the specific format of the cognitive operation is similar across both groups. Indeed, if the lack of vision does not preclude the optimal development of various numerical skills (Dormal et al., 2016b; Castronovo, 2014; Crollen and Collignon, 2016), some qualitative properties of numerical representations do seem to critically depend on early visual experience. For example, congenital blindness changes the nature of the reference frame in which the spatial processing of numbers occurs: while sighted and late blind participants associate numbers to an external frame of reference, $\mathrm{CB}$ individuals principally rely on an association between numbers and an egocentric coordinate system (Crollen et al., 2013). Blindness also alters the typical development of finger-counting, a procedure often used by sighted individuals while learning basic addition and subtraction operations (Crollen et al., 2011, 2014). Given that congenital blindness affects the use of an external visuo-spatial frame of reference and the implementation of finger-counting, it is possible that the common regions involved in numerical processing in both groups rely on distinct representational formats in $\mathrm{CB}$ and sighted individuals. It will therefore be important for future studies to assess whether the representations of the numerical information embedded in those brain circuits are truly independent of visual experience or if visual experience influences the format of these representations despite overlapping activations between groups.

Interestingly, some studies demonstrated that mathematical abilities do not correlate with specific computations: size and density sensitivity do not correlate with math, in contrast to visual numerosity (Anobile et al., 2016, 2018). Similarly, average size (Castaldi et al., 2018), line length (Cappelletti et al., 2014; De Visscher et al., 2017) and area (Iuculano et al., 2008) are not impaired by dyscalculia, a learning deficit affecting number processing. The evidence that numerical concepts can be acquired in $\mathrm{CB}$ raise the question as to whether these perceptual factors that link to the development of arithmetic abilities in the sighted are independent from visual experience or whether, in contrast, CB people rely on separate perceptual processes in order to develop adequate arithmetic abilities through separate cognitive mechanisms (Dormal et al., 2016b; Crollen et al., 2017). 


\section{Conclusions}

Arithmetic represents an interesting framework to examine whether the recruitment of the occipital cortex in CB individuals by higher-level cognitive functions may be linked to the intrinsic computational role of specific brain regions. Indeed, separate arithmetic operations rely on separate brain networks depending on the computational principle they rely on. While subtraction principally engages spatial strategies and a dorsal parieto-frontal network, multiplication principally relies on memory retrieval and temporo-parietal regions. In this paper, we suggested that subtraction (but not multiplication) may find a neural niche in dorsal occipital regions since these brain areas maintain a privileged role in processing spatial relationship in $\mathrm{CB}$ individuals.

\section{Acknowledgments}

The authors are grateful to Giulia Dormal for her help in implementing the design of this study. This research and the authors were supported by the Canada Research Chair Program (FL), the Canadian Institutes of Health Research (FL), the Belgian National Funds for Scientific Research (OC, MPN), a WBI.World grant (VC), the European Union's Horizon 2020 research and innovation program under the Marie Sklodowska-Curie grant agreement No 700057 (VC) and the 'MADVIS' European Research Council starting grant (ERC-StG 337573) attributed to OC. OC is a research associate at the Fond National de la recherche scientifique de Belgique.

\section{Appendix A. Supplementary data}

Supplementary data to this article can be found online at https://doi. org/10.1016/j.neuroimage.2018.11.034.

\section{Competing financial interests}

The authors declare that they do not have any financial interests that could be construed to have influenced their paper.

\section{References}

Abboud, S., Maidenbaum, S., Dehaene, S., Amedi, A., 2015. A number-form area in the blind. Nat. Commun. 6 (6026), 1-9.

Amedi, A., Hofstetter, S., MAidenbaum, S., Heimler, B., 2017. Task selectivity as a comprehensive principle for brain organization. Trends Cognit. Sci. 5, 307-310.

Amedi, A., Raz, N., Pianka, P., Malach, R., Zohary, E., 2003. Early "visual" cortex activation correlates with superior verbal memory performance in the blind. Nat. Neurosci. 6 (7), 758-766.

Anobile, G., Arrighi, R., Castaldi, E., Grassi, E., Pedonese, L., Moscoso, P.A.M., Burr, D.C., 2018. Spatial but not temporal numerosity thresholds correlate with formal math skills in children. Dev. Psychol. 54 (3), 458-473. https://doi.org/10.1037/ dev0000448.

Anobile, G., Castaldi, E., Turi, M., Tinelli, F., Burr, D.C., 2016. Numerosity but not texturedensity discrimination correlates with math ability in children. Dev. Psychol. 52 (8), 1206-1216. https://doi.org/10.1037/dev0000155.

Bavelier, D., Neville, H.J., 2002. Cross-modal plasticity: where and how? Nat. Rev. Neurosci. 3, 443-452.

Bedny, M., 2017. Evidence from blindness for a cognitively pluripotent cortex. Trends Cognit. Sci. 21 (9), 637-648

Bedny, M., Pascual-Leone, A., Dodell-Feder, D., Fedorenko, F., Saxe, R., 2011. Language processing in the occipital cortex of congenitally blind adults. Proc. Natl. Acad. Sci. U.S.A. 108 (11), 4429-4434.

Behzadi, Y., Restom, K., Liau, J., Liu, T.T., 2007. A component based noise correction method (CompCor) for BOLD and perfusion based fMRI. Neuroimage 37 (1), 90-101.

Bi, Y., Wang, X., Caramazza, A., 2016. Object domain and modality in the ventral visual pathway. Trends Cognit. Sci. 20 (4), 282-290.

Büchel, C., Price, C., Frackowiak, R.S.J., Friston, K., 1998. Different activation patterns in the visual cortex of late and congenitally blind subjects. Brain 121 (3), 409-419.

Burr, D.C., Annobile, G., Arrighi, R., 2018. Psychophysical evidence for the number sense. Philos. Trans. R. Soc. Lond. B Biol. Sci. 373 (1740) https://doi.org/10.1098/ rstb.2017.0045.

Burton, H., Snyder, A.Z., Raichle, M.E., 2014. Resting state functional connectivity in early blind humans. Front. Syst. Neurosci. 8, 51.

Campbell, J.I.D., Timm, J.C., 2000. Adult's strategy choices for simple addition: effects of retrieval interference. Psychonomic Bull. Rev. 7 (4), 692-699.
Campbell, J.I.D., Xue, Q., 2001. Cognitive arithmetic across cultures. J. Exp. Psychol. Gen. 130 (2), 299-315.

Cappelletti, M., Chamberlain, R., Freeman, E.D., Kanai, R., 2014. Commonalities for numerical and continuous quantity skills at temporo-parietal junction. J. Cognit. Neurosci. 26 (5), 986-999. https://doi.org/10.1162/jocn_a_00546.

Castaldi, E., Mirassou, A., Dehaene, S., Piazza, M., Eger, E., 2018. Asymmetrical interference between number and item size perception provide evidence for a domain specific impairment in dyscalculia. bioRxiv, 332155. https://doi.org/10.1101/ 332155.

Castronovo, J., 2014. Numbers in the dark: numerical cognition and blindness. In: Cohen Kadosh, R., Dowker, A. (Eds.), The Oxford Handbook of Numerical Cognition. Oxford University Press, Oxford, pp. 787-807.

Cecchetti, L., Kupers, R., Ptito, M., Pietrini, P., Ricciradi, E., 2016. Are supramodality and cross-modal plasticity the yin and yang of brain development? From blindness to rehabilitation. Front. Syst. Neurosci. 10, 89.

Chochon, F., Cohen, L., van de Moortele, P.F., Dehaene, S., 1999. Differential contributions of the left and right inferior parietal lobules to number processing. J. Cognit. Neurosci. 11 (6), 617-630.

Collignon, O., Dormal, G., Lepore, F., 2012. Building the brain in the dark: functional and specific crossmodal reorganization in the occipital cortex of blind individuals. In: Steeves, J.K.E., Harris, L.R. (Eds.), Plasticity in Sensory Systems. Cambridge University Press, pp. 73-96.

Collignon, O., Lassonde, M., Lepore, F., Bastien, D., Veraart, C., 2007. Functional cerebral reorganization for auditory spatial processing and auditory substitution of vision in early blind subjects. Cerebr. Cortex 17 (2), 457-465.

Collignon, O., Vandewalle, G., Voss, P., Albouy, G., Charbonneau, G., Lassonde, M., Lepore, F., 2011. Functional specialization for auditory-spatial processing in the occipital cortex of congenitally blind humans. Proc. Natl. Acad. Sci. U.S.A. 108 (11), 4435-4440.

Collignon, O., Voss, P., Lassonde, M., Lepore, F., 2009. Cross-modal plasticity for the spatial processing of sounds in visually deprived subjects. Exp. Brain Res. 192 (3), 343-358.

Cooney, J.B., Swanson, H.L., Ladd, S.F., 1988. Acquisition of mental multiplication skill: evidence for the transition between counting and retrieval strategies. Cognit. InStruct. 5, 323-345.

Corbetta, M., Shulman, G.L., Miezin, F.M., Petersen, S.E., 1995. Superior parietal cortex activation during spatial attention shifts and visual feature conjunction. Science 270 (5237), 802-805.

Crollen, V., Collignon, O., 2016. Cognition numérique : que peuvent nous apprendre les personnes non-voyantes? Med. Sci. 32, 1097-1102.

Crollen, V., Collignon, O., Noël, M.P., 2017. Visuo-spatial processes as a domain-general factor of numerical development in atypical populations. Journal of Numerical Cognition 3 (2). https://doi.org/10.5964/jnc.v3i2.68.

Crollen, V., Dormal, G., Seron, X., Lepore, F., Collignon, O., 2013. Embodied numbers: the role of vision in the development of number-space interactions. Cortex 49, 276-283.

Crollen, V., Mahe, R., Collignon, O., Seron, X., 2011. The role of vision in the development of finger-number interactions: finger-counting and finger-montring in blind children. J. Exp. Child Psychol. 109, 525-539.

Crollen, V., Noël, M.P., Seron, X., Mahau, P., Lepore, F., Collignon, O., 2014. Visual experience influences the interactions between fingers and numbers. Cognition 133, 91-96.

Damarla, S.R., Cherkassky, V.L., Just, M.A., 2016. Modality-independent representations of small quantities based on brain activation patterns. Hum. Brain Mapp. 37 (4) 1296-1307.

Dehaene, S., Cohen, L., 1997. Cerebral pathways for calculation: double dissociation between rote verbal and quantities knowledge of arithmetic. Cortex 33 (2), 219-250.

Dehaene, S., Cohen, L., 2007. Cultural recycling of cortical maps. Neuron 56 (2), 384-398.

Delazer, M., Benke, T., 1997. Arithmetic facts without meaning. Cortex 33 (4), 697-710.

De Visscher, A., Noël, M.P., Pesenti, M., Dormal, V., 2017. Developmental dyscalculia in adults : beyond numerical magnitude impairment. J. Learn. Disabil. https://doi.org/ 10.1177/0022219417732338, 22219417732338.

Dormal, G., Collignon, O., 2011. Functional selectivity in sensory-deprived cortices. J. Neurophysiol. 105 (6), 2627-2630.

Dormal, G., Lepore, F., Collignon, O., 2012. Plasticity of the dorsal « spatial » stream in visually deprived individuals. Neural Plast. 2012, 687659.

Dormal, G., Rezk, M., Yakobov, E., Lepore, F., Collignon, O., 2016a. Auditory motion in the sighted and blind: early visual deprivation triggers a large-scale imbalance between auditory and «visual » brain regions. Neuroimage 134, 630-644.

Dormal, V., Crollen, V., Baumans, C., Lepore, F., Collignon, O., 2016b. Early but not late blindness leads to enhanced arithmetic and working memory abilities. Cortex 83, 212-221.

Eger, E., Sterzer, P., Russ, M.O., Giraud, A.L., Kleinschmidt, A., 2003. A supramodal number representation in human intraparietal cortex. Neuron 37 (4), 719-725.

Gougoux, F., Zatorre, R.J., Lassonde, M., Voss, P., Lepore, F., 2005. A functional neuroimaging study of sound localization: visual cortex activity predicts performance in early-blind individuals. PLoS Biol. 3, e27.

Grabner, R.H., Ansari, D., Reishofer, G., Stern, E., Ebner, F., Neuper, C., 2007. Individual differences in mathematical competence predict parietal brain activation during mental calculation. Neuroimage 38, 346-356.

Hannagan, T., Amedi, A., Cohen, L., Dehaene-Lambertz, G., Dehaene, S., 2015. Origins of the specialization for letters and numbers in ventral occipitotemporal cortex. Trends Cognit. Sci. 19 (7), 374-382.

Hasson, U., Andric, M., Atilgan, H., Collignon, O., 2016. Congenital blindness is associated with large-scale reorganization of anatomical networks. Neuroimage 128 , $362-372$. 
Hecht, S.A., 1999. Individual solution processes while solving addition and multiplication math facts in adults. Mem. Cognit. 27 (6), 1097-1107.

Heimler, B., Striem-Amit, E., Amedi, A., 2015. Origins of task-specific sensoryindependent organization in the visual and auditory brain: neuroscience evidence, open questions and clinical implications. Curr. Opin. Neurobiol. 35, 169-177.

Iuculano, T., Tang, J., Hall, C.W., Butterworth, B., 2008. Core information processing deficits in developmental dyscalculia and low numeracy. Dev. Sci. 11 (5), 669-680. https://doi.org/10.1111/j.1467-7687.2008.00716.x.

Jiang, F., Stecker, G.C., Boynton, G.M., Fine, I., 2016. Early blindness results in developmental plasticity for auditory motion processing within auditory and occipital cortex. Front. Hum. Neurosci. 10, 324.

Kanjlia, S., Lane, C., Feigenson, L., Bedny, M., 2016. Absence of visual experience modifies the neural basis of numerical thinking. Proc. Natl. Acad. Sci. U. S. A. 113 (40), 11172-11177.

Katz, C., Hoesterey, H., Knops, A., 2017. A role for attentional reorienting during approximate multiplication and division. J. Numer. Cogn. 3, 246-269. https:// doi.org/10.5964/jnc.v3i2.62.

Katz, C., Knops, A., 2014. Operational momentum in multiplication and division? PLoS One 9, e104777. https://doi.org/10.1371/journal.pone.0104777.

Knops, A., Thirion, B., Hubbard, E.M., Michel, V., Dehaene, S., 2009a. Recruitment of an area involved in eye movements during mental arithmetic. Science 324, 1583-1585.

Knops, A., Viarouge, A., Dehaene, S., 2009b. Dynamic representations underlying symbolic and non-symbolic calculation: evidence from the operational momentum effect. Atten. Percept. Psychophys. 71 (4), 803-821.

Lacey, S., Stilla, R., Deshpande, G., Zhao, S., Stephens, C., McCormick, K., Kemmerer, D., Sathian, K., 2017. Engagement of the left extrastriate body area during body-part metaphor comprehension. Brain Lang. 166, 1-18. https://doi.org/10.1016/ j.bandl.2016.11.004.

Lacey, S., Stilla, R., Sathian, K., 2012. Metaphorically feeling: comprehending textural metaphors activates somatosensory cortex. Brain Lang. 120 (3), 416-421. https:// doi.org/10.1016/j.bandl.2011.12.016.

Lane, C., Kanjlia, S., Omaki, A., Bedny, M., 2015. "Visual" cortex of congenitally blind adults responds to syntactic movement. J. Neurosci. 35 (37), 12859-12868.

Lasne, G., Piazza, M., Dehaene, S., Kleinschmidt, A., Eger, E., 2018. Discriminability of numerosity-evoked fMRI activity patterns I human intra-parietal cortex reflects behavioral numerical acuity. Cortex. https://doi.org/10.1016/j.cortex.2018.03.008 pii: S0010-9452(18)30089-3.

Lee, K.M., 2000. Cortical areas differentially involved in multiplication and subtraction: a functional magnetic resonance imaging study and correlation with a case of selective acalculia. Ann. Neurol. 48 (4), 657-661.

LeFevre, J., Bisanz, J., Daley, K.E., Buffone, L., Greenham, S.L., Sadesky, G.S., 1996. Multiple routes to solution of single-digit multiplication problems. J. Exp. Psychol. Gen. 125 (3), 284-306.

Li, M., Liu, D., Li, M., Dong, W., Huang, Y., Chen, Q., 2018. Addition and subtraction but not multiplication and division cause shifts of spatial attention. Front. Hum. Neurosci. 12, 183. https://doi.org/10.3389/fnhum.2018.00183.

Masson, N., Pesenti, M., 2014. Attentional bias induced by solving simple and complex addition and subtraction problems. Q. J. Exp. Psychol. 67 (8), 1514-1526.

Mathieu, R., Epinat-Duclos, J., Sigovan, M., Breton, A., Cheylus, A., Fayol, M., Thevenot, C., Prado, J., 2018a. What's behind a "+" sign? Perceiving an arithmetic operator recruits brain circuits for spatial orienting. Cerebr. Cortex 28, 1673-1684.

Mathieu, R., Epinat-Duclos, J., Léone, J., Fayol, M., Thevenot, C., Prado, J., 2018b. Hippocampal spatial mechanisms relate to the development of arithmetic symbol processing in children. Dev Cogn Neurosci 30, 324-332.

Mathieu, R., Gourjon, A., Couderc, A., Thevenot, C., Prado, J., 2016. Running the number line: rapid shifts of attention in single-digit arithmetic. Cognition 146, 229-239. https://doi.org/10.1016/j.cognition.2015.10.002.

Mattioni, S., Rezk, M., Battal, C., Vadlamudi, J., Collignon, O., 2018. The balanced act of crossmodal and intramodal plasticity: Enhanced representation of auditory categories in the occipital cortex of early blind people links to reduced temporal coding. J. Vis. 18 (10), 554.

McCrink, K., Dehaene, S., Dehaene-Lambertz, G., 2007. Moving along the mental number line: operational momentum in nonsymbolic arithmetic. Percept. Psychophys. 69 (8), 1324-1333.

Menon, V., Rivera, S.M., White, C.D., Glover, G.H., Reiss, A.L., 2000. Dissociating prefrontal and parietal cortex activation during arithmetic processing. Neuroimage 12 (4), 357-365.

Mizuhara, H., Wang, L.Q., Kobayashi, K., Yamaguchi, Y., 2005. Long-range EEG phase synchronization during an arithmetic task indexes a coherent cortical network simultaneously measured by fMRI. Neuroimage 27 (3), 553-563.

Nieder, A., 2012. Supramodal numerosity selectivity of neurons in primate prefrontal and posterior parietal cortices. Proc. Natl. Acad. Sci. Unit. States Am. 109 (29), 11860-11865.

Nieder, A., 2016. The neuronal code for number. Nat. Rev. Neurosci. 17 (6), 366-382.

Pelland, M., Orban, P., Dansereau, C., Lepore, F., Bellec, P., Collignon, O., 2017. Statedependent modulation of functional connectivity in early blind individuals. Neuroimage $147,532-541$.
Penny, W., Friston, K., Ashburner, J., Kiebel, S., Nichols, T., 2008. Statistical Parametric Mapping: the Analysis of Functional Brain Images. Academic press.

Pesenti, M., Seron, X., van der Linden, M., 1994. Selective impairment as evidence for mental organization of arithmetic facts: BB, a case of preserved subtraction? Cortex $30,661-671$.

Piazza, M., Pinel, P., Le Bihan, D., Dehaene, S., 2007. A magnitude code common to numerosities and number symbols in human intraparietal cortex. Neuron 53, 293-305.

Pinhas, M., Fischer, M., 2008. Mental movements without magnitude? A study of spatial biases in symbolic arithmetic. Cognition 109, 408-415.

Poirier, C., Collignon, O., Scheiber, C., Renier, L., Vanlierde, A., Tranduy, D., Veraart, C., De Volder, A.G., 2006. Auditory motion perception activates visual motion areas in early blind subjects. Neuroimage 31 (1), 279-285.

Power, J.D., Barnes, K.A., Snyder, A.Z., Schlaggar, B.L., Petersen, S.E., 2012. Spurious but systematic correlations in functional connectivity MRI networks arise from subject motion. Neuroimage 59 (3), 2142-2154.

Prado, J., Mutreja, R., Booth, J.R., 2014. Developmental dissociation in the neural responses to simple multiplication and subtraction problems. Dev. Sci. 17 (4), $537-552$.

Prado, J., Mutreja, R., Zhang, H., Mehta, R., Desroches, A.S., Minas, J.E., Booth, J.R., 2011. Distinct representations of subtraction and multiplication in the neural systems for numerosity and language. Hum. Brain Mapp. 32, 1932-1947.

Reich, L., Szwed, M., Cohen, L., Amedi, A., 2011. A ventral visual stream reading center independent of visual experience. Curr. Biol. 21 (5), 363-368.

Ricciardi, E., Tozzi, L., Leo, A., Pietrini, P., 2014. Modality-dependent cross-modal functional reorganization following congenital visual deprivation within occipital areas: a meta-analysis of tactile and auditory studies. Multisensory Res. 27 (3-4), $247-262$.

Ricciardi, E., Vanello, N., Sani, L., Gentili, C., Scilingo, E.P., Landini, L., Guazzelli, M., Bicchi, A., Haxby, J.V., Pietrini, P., 2007. The effect of visual experience on the development of functional architecture in hMT +. Cerebr. Cortex 17 (12), 2933-2939.

Rickard, T.C., Romero, S.G., Basso, G., Wharton, C., Flitman, S., Grafman, J., 2000. The calculating brain: an fMRI study. Neuropsychologia 38 (3), 325-335.

Röder, B., Stock, O., Bien, S., Neville, H., Rösler, F., 2002. Speech processing activates visual cortex in congenitally blind humans. Eur. J. Neurosci. 16 (5), 930-936.

Sadato, N., Pascual-Leone, A., Grafman, J., Ibanez, V., Deiber, M.P., Dold, G., Hallett, M., 1996. Activation of the primary visual cortex by Braille reading in blind subjects. Nature 380, 526-528.

Satterthwaite, T.D., Wolf, D.H., Loughead, J., Ruparel, K., Elliott, M.A., Hakonarson, H., Gur, R.C., Gur, R.E., 2012. Impact of in-scanner head motion on multiple measures of functional connectivity: relevance for studies of neurodevelopment in youth. Neuroimage 60 (1), 623-632.

Siegler, R.S., Shrager, J., 1984. Strategy choices in addition and subtraction: how do children know what to do? In: Sophian, C. (Ed.), The Origins of Cognitive Skills. Erlbaum, Hillsdale, pp. 229-293.

Sladky, R., Friston, K.J., Tröstl, J., Cunnington, R., Moser, E., Windischberger, C., 2011. Slice timing effects and their correction in functional MRI. Neuroimage 58 (2), 588-594.

Talairach, J., Tournoux, P., 1988. Co-planar Stereotaxic Atlas of the Human Brain. Thieme, New York.

van Ackeren, M.J., Barbero, F.M., Mattioni, S., Bottini, R., Collignon, O., 2017. Neuronal populations in the occipital cortex of the blind synchronize to the temporal dynamics of speech. BioRxiv, 186338. https://doi.org/10.1101/186338.

Van Dijk, K.R., Sabuncu, M.R., Buckner, R.L., 2012. The influence of head motion on intrinsic functional connectivity MRI. Neuroimage 59 (1), 431-438.

van Harskamp, N.J., Cipolotti, L., 2001. Selective impairments for addition, subtraction and multiplication. Implications for the organization of arithmetical facts. Cortex 37 (3), 363-388.

Weeks, R., Horwitz, B., Aziz-Sultan, A., Tian, B., Wessinger, C.M., Cohen, L.G., Hallett, M., Rauschecker, J.P., 2000. A positron emission tomographic study of auditory localization in the congenitally blind. J. Neurosci. 20 (7), 2664-2672.

Whitfield-Gabrieli, S., Nieto-Castanon, A., 2012. Conn: a functional connectivity toolbox for correlated and anticorrelated brain networks. Brain Connect. https://doi.org/ 10.1089/brain.2012.0073.

Wu, S., Chang, T.T., Majid, A., Caspers, S., Eickhoff, S.B., Menon, V., 2009. Functional heterogeneity of inferior parietal cortex during mathematical cognition assessed with cytoarchitectonic probability maps. Cerebr. Cortex 19 (12), 2930-2945.

Yantis, S., Serences, J.T., 2003. Cortical mechanisms of space-based and object-based attentional control. Curr. Opin. Neurobiol. 13 (2), 187-193.

Zago, L., Pesenti, M., Mellet, E., Crivello, F., Mazoyer, B., Tzourio-Mazoyer, N., 2001. Neural correlates of simple and complex mental calculation. Neuroimage 13 (2), 314-327.

Zhou, X., Chen, C., Zang, Y., Dong, Q., Chen, C., Qiao, S., Gong, Q., 2007. Dissociated brain organization for single digit addition and multiplication. Neuroimage 35 (2), 871-880. 